\title{
Theory of Compaction Bands in Porous Rock
}

\author{
K. A. Issen* \\ J. W. Rudnicki \\ Department of Civil Engineering, Northwestern University, Evanston, Illinois, USA
}

\author{
Camera-ready Copy for \\ Physics and Chemistry of the Earth \\ Manuscript-No. SE35-013 \\ Offset requests to: \\ K. A. Issen \\ Clarkson University \\ Potsdam, New York
}

${ }^{*}$ Present address: Department of Mechanical and Aeronautical Engineering, Clarkson University, Potsdam, New York, USA 
Journal: Physics and Chemistry of the Earth

MS No.: SE35-013

First author: K. A. Issen

\title{
Theory of Compaction Bands in Porous Rock
}

\author{
K. A. Issen* \\ J. W. Rudnicki \\ Department of Civil Engineering, Northwestern University, Evanston, Illinois, USA \\ ${ }^{*}$ Present address: Department of Mechanical and Aeronautical Engineering, Clarkson University, Potsdam, New \\ York, USA
}

Received June 15, 2000 - Accepted September 22, 2000

\begin{abstract}
Compaction bands are narrow planar zones of localized purely compressive (without shear) deformation that form perpendicular to the most compressive principal stress. Such bands have been observed in high porosity rocks in the laboratory and in the field. Because compaction presumably decreases permeability, these bands can act as barriers to flow within reservoirs. Reexamination of the results of Rudnicki and Rice (J. Mech. Phys. Solids, 1975) for shear localization, with corrections by Perrin and Leblond (J. Appl. Mech., 1993), reveals that they admit solutions for compaction bands in a range of parameters that is representative of porous rock. Solutions for compaction bands are possible when the inelastic volume deformation is compactive and is associated with a "cap" on the yield surface. The expression for the critical hardening modulus (related to the slope of the shear stress vs. shear strain curve at constant mean stress) at which compaction bands are predicted to form differs from that for shear localization. For parameters representative of porous rock, axisymmetric compression is the most favorable deviatoric stress state for formation of compaction bands. Comparison of conditions for shear localization and compaction band formation suggests that either may occur depending on the stress path and magnitude of the confining stress.
\end{abstract}

\section{Introduction}

Compaction bands are narrow, planar zones of material that is denser than that surrounding. Mollema and Antonellini (1996) have reported field observations of compaction bands in porous aeolian sandstone. The material within the bands has apparently been compacted without shear and the bands appear to be oriented perpendicular to the maximum compressive prin-

Correspondence to: K. A. Issen cipal stress. ? has summarized laboratory observations of compaction bands.

Compaction bands may significantly affect fluid flow in situ. Because the material within the bands is more dense, it presumably has a lower permeability than surrounding material. Hence, bands may constitute barriers to fluid flow. The presence of compaction bands in highly porous hydrocarbon reservoirs could adversely affect attempts to inject or extract fluids for energy storage or production. The denser material within the bands may also be stronger than the surrounding material. Thus, the presence of bands will also affect the mechanical strength of the formation. Because the bands are localized structures, they will be difficult to detect from the surface by geophysical methods. For these reasons, understanding the stress states and material properties that are conducive to compaction band formation is important.

How do compaction bands form? Why should the deformation localize in a planar zone? ? addressed these questions within the framework of bifurcation theory used by Rudnicki and Rice (1975) to study conditions for the formation of shear bands in pressure-sensitive, dilatant materials. ? showed that compaction bands were possible for porous rocks for which predominantly hydrostatic compressive stress states cause inelastic volume decrease. The conditions obtained by ? for band formation were restrictive. However, by extending the approach of ? and by reexamining the Rudnicki and Rice (1975) analysis, Issen and Rudnicki (2000) identified less restrictive conditions for compaction band formation. Although Rudnicki and Rice (1975) had identified this possibility, these solutions were not discussed because of their focus on low porosity, dilatant rocks. By incorporating a correction to the original Rudnicki and Rice (1975) analysis, which was pointed out by Perrin and Leblond (1993), Issen and Rudnicki (2000) showed that compaction bands were the preferred non-uniform solution (compared with shear bands) for a range of ma- 
terial parameters that is representative of high porosity rock. This paper continues the analysis of Issen and Rudnicki (2000). In particular, we examine a wider framework of constitutive relations, which includes the possibility of a yield surface cap (shear stress required for further inelastic deformation decreases with increasing compressive mean stress). We also discuss the consequences of loading at a corner, which occurs if the cap and shear yield surfaces do not meet smoothly.

\section{Bifurcation Approach to Localization}

Building upon earlier analyses of Hadamard (1903), Mandel (1966), Thomas (1961) and Hill (1962), Rudnicki and Rice (1975) showed that localized deformation in a planar band with normal $\mathbf{n}$ is an alternative to (bifurcation from) homogeneous deformation if a nontrivial solution exists to the following eigenvalue problem:

$$
\left\{n_{i} L_{i j k l} n_{l}\right\} g_{k}=0
$$

A non-trivial solution for the $g_{k}$ is possible only when

$$
\operatorname{det}\left\{n_{i} L_{i j k l} n_{l}\right\}=0
$$

In (1) and (2), $\mathbf{L}$ is the tensor appearing in an incrementally linear relation between the stress-rate $\dot{\sigma}$ and the strain-rate $\dot{\varepsilon}$

$$
\dot{\sigma}_{i j}=L_{i j k l} \dot{\varepsilon}_{k l}
$$

The components of $\mathbf{L}$ are symmetric with respect to interchange of the first two indices and the last two, but not necessarily with respect to interchange of the first pair with the last pair. (The relation (3) is properly written for work-conjugate stress and strain measures and an objective stress rate (Hill , 1968). But the effect of this refinement on the localization condition is typically small (Rudnicki and Rice, 1975) and, for simplicity, we neglect it here.)

The condition (1) is the result of the requirements that the non-uniform field be in equilibrium and that the velocity field be continuous at the instant of band formation or localization. Equilibrium is expressed by the condition that the traction-rates be continuous across the band boundary. A continuous velocity field requires that the difference in the strain-rates inside and outside of the band has the form of the symmetric part of the tensor with components $n_{i} g_{j}$, where the $g_{j}$ are functions of distance across the band. A recent analysis of Bésuelle (2000) has shown that the localized zone corresponds to a shear band when the vector $\mathbf{g}$ has a component parallel to the plane of the band and may, in addition, be dilating or compacting depending on whether $\mathbf{n} \cdot \mathbf{g}$ is positive or negative. For a compaction band $\mathbf{g}$ is in the direction of $\mathbf{n}$ and opposite to it for a dilation band.
The condition (2) relates the constitutive properties and the band directions for which localized solutions are possible. Obviously, the possibility that (2) can be met for some orientation depends strongly on the nature of the constitutive relation for homogeneous deformation that defines the components of $\mathbf{L}$.

\section{Constitutive Relation}

Experimental testing of rocks has shown that there is a set of stress states for which the response can be described as elastic. For stress states on the boundary of this region, the yield surface, the deformation is nonelastic and the size and shape of this elastic region may evolve with non-elastic deformation. For simplicity, we assume that the yield surface depends on stress only through the first and second invariants (or equivalently the first invariant of the stress and the second invariant of the deviatoric stress). The first invariant is denoted by $\sigma=-(1 / 3) \sigma_{k k}$, where the repeated subscript denotes summation and $\sigma_{k k}$ is assumed to be positive in tension. The second invariant of the deviatoric stress is given by $\tau=\sqrt{(1 / 2) s_{i j} s_{i j}}$, where the $s_{i j}=\sigma_{i j}-(1 / 3) \delta_{i j} \sigma_{k k}$ are components of the deviatoric stress and $\delta_{i j}=1$ if $i=j$, $\delta_{i j}=0$ if $i \neq j$. If inelastic deformation is assumed to depend on stress only through $\tau$ and $\sigma$, then the yield condition can be written as

$$
F\left(\tau, \sigma, \alpha_{\kappa}\right)=0
$$

where the $\alpha_{\kappa}$ denote a set of variables that keep track of the history of inelastic deformation. In the simplest cases, these may be the inelastic strains themselves or some scalar combination of them. More generally, they may be internal variables such as microcrack density, dislocation density, or porosity. For fixed $\alpha_{\kappa}$ the yield condition describes a surface in the $\tau$ vs. $\sigma$ plane (see Figure 1) with slope $\mu=-(\partial F / \partial \sigma) /(\partial F / \partial \tau)$. When the stress state is on the yield surface, the inelastic strain increments are assumed to be expressed in terms of a plastic potential $\Gamma\left(\tau, \sigma, \alpha_{\kappa}\right)$ :

$$
d \varepsilon_{i j}^{p}=d \lambda \frac{\partial \Gamma}{\partial \sigma_{i j}}=d \lambda\left\{\frac{s_{i j}}{2 \tau} \frac{\partial \Gamma}{\partial \tau}-\frac{1}{3} \frac{\partial \Gamma}{\partial \sigma} \delta_{i j}\right\}
$$

where $d \lambda$ is a non-negative scalar parameter. An implication of this form is that the increment of inelastic volume strain is connected to the increment of inelastic equivalent shear strain, $d \gamma^{p}=\left(2 d e_{i j}^{p} d e_{i j}^{p}\right)^{1 / 2}\left(d e_{i j}^{p}\right.$ is the deviatoric part of $\left.d \varepsilon_{i j}^{p}\right)$, by $d \varepsilon_{k k}^{p}=\beta d \gamma^{p}$, where $\beta=-(\partial \Gamma / \partial \sigma) /(\partial \Gamma / \partial \tau)$ is called the dilatancy factor.

Because the $\alpha_{\kappa}$ must change with inelastic deformation so that the stress state remains on the yield surface the following consistency condition must be satisfied:

$$
d F=\frac{\partial F}{\partial \tau} d \tau+\frac{\partial F}{\partial \sigma} d \sigma+\frac{\partial F}{\partial \alpha_{\kappa}} d \alpha_{\kappa}=0
$$




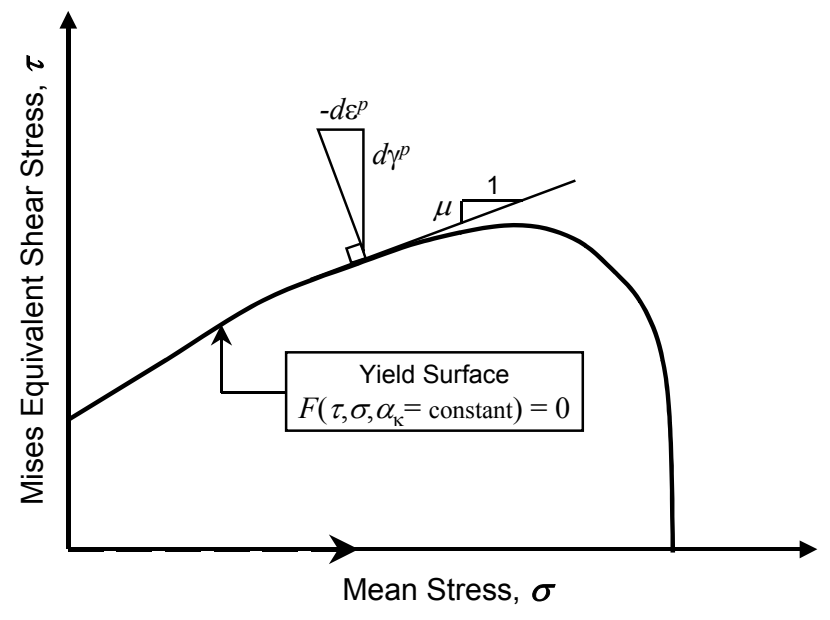

Fig. 1. For fixed $\alpha_{\kappa}$, the yield function, $F=0$, describes a yield surface (corresponding to stress states that cause non-elastic deformation) in the $\tau-\sigma$ plane. A possible yield surface for high porosity rock (closed on the $\sigma$ - axis) is shown.

For a rate-independent constitutive relation, changes in the $\alpha_{\kappa}$ are related linearly to $d \varepsilon_{i j}^{p}$ :

$$
d \alpha_{\kappa}=\Phi_{i j}^{\kappa}\left(\tau, \sigma, \alpha_{\xi}\right) d \varepsilon_{i j}^{p}
$$

Substituting (5) into (7), the result into (6), solving for $d \lambda$ and substituting the result into (5) yields the following expression for the plastic strain increments

$$
d \varepsilon_{i j}^{p}=\frac{1}{H}\left\{\frac{s_{i j}}{2 \tau} \frac{\partial \Gamma}{\partial \tau}-\frac{1}{3} \frac{\partial \Gamma}{\partial \sigma} \delta_{i j}\right\}\left\{\frac{\partial F}{\partial \tau} d \tau+\frac{\partial F}{\partial \sigma} d \sigma\right\}
$$

where

$$
H=-\frac{\partial F}{\partial \alpha_{\kappa}} \Phi_{k l}^{\kappa}\left(\tau, \sigma, \alpha_{\xi}\right) \frac{\partial \Gamma}{\partial \sigma_{k l}}
$$

is a plastic hardening modulus.

In their study of conditions for shear localization, Rudnicki and Rice (1975) used this framework with $\partial F / \partial \tau=\partial \Gamma / \partial \tau=1$. Holcomb and Rudnicki (2000) used the same framework with $\gamma^{p}$ as the sole parameter $\left(\alpha_{\kappa}\right)$ characterizing the history of plastic deformation to describe a suite of axisymmetric compression tests at confining pressures from 0 to $100 \mathrm{MPa}$ on Tennessee Marble. Because Rudnicki and Rice (1975) focused on shear localization in low porosity, dilatant rocks, they restricted attention to values of $\mu$ (local slope of yield surface) and $\beta$ (dilatancy factor) that were positive. In this case, the yield surface is not closed on the hydrostatic $(\sigma)$ axis and no inelastic deformation is caused by purely hydrostatic stress (at least in the pressure range of interest). By considering negative values of $\mu$ and $\beta$ the formulation can be applied to the case where the yield surface is closed on the $\sigma$ axis and the inelastic volume strain is compactive, as appropriate for high porosity, compacting rocks. For such rocks, using $\gamma^{p}$ as the sole parameter characterizing inelastic deformation, as assumed by Holcomb and Rudnicki (2000) is unlikely to be suitable. At a minimum, it is expected that the yield surface would evolve with the inelastic volume strain or inelastic portion of the porosity.

The framework used by Rudnicki and Rice (1975) does, however, break down for purely hydrostatic stressing. By symmetry $\partial F / \partial \tau$ (and likely $\partial \Gamma / \partial \tau$ ) must vanish for $\tau=0$ (the slope of the yield surface is vertical). Furthermore, microstructural observations (Menéndez et al. , 1996) have indicated that the mechanisms of inelastic deformation for low porosity dilatant rocks (microcrack creation and growth) differ from those of high porosity, compacting rock (pore collapse, grain disaggregation and grain crushing). Consequently, it is plausible that these differing mechanisms are reflected at the macroscopic level by two independent yield surfaces. This observation has been implemented by introducing a "cap" on the yield surface. The "cap" model (often depicted as approximately a quarter ellipse intersecting the mean stress axis perpendicularly) was apparently first introduced by DiMaggio and Sandler (1971) for soils, but has been widely used to describe the compactive behavior of high porosity rock (Wong et al. , 1992; ?; Fossum and Fredrich , 2000). Issen (2000) used a specialized version of the constitutive framework discussed above to represent the cap yield surface: $\partial F / \partial \sigma=$ $\partial \Gamma / \partial \sigma=1$, with $\varepsilon^{p}$ as the sole parameter $\left(\alpha_{\kappa}\right)$ characterizing the history of plastic deformation. This formulation accommodates inelastic volume strain due to hydrostatic pure compression.

Aydin and Johnson (1983) accounted for inelastic volume strain due to hydrostatic compression by adding a term to the Rudnicki and Rice (1975) constitutive relation. The modified relation is obtained by replacing $K$ (the elastic bulk modulus), with $K^{*}=K k /(K+k)$, where $k$ (the "bulk" hardening modulus) is the slope of the mean stress - inelastic volume strain curve at constant shear stress. Issen (2000) showed that this change is equivalent to the activation of a second yield surface - a flat cap perpendicular to the mean stress axis, such that both the flat cap and shear yield surfaces are active and form a vertex (i.e., join with unequal slopes).

\section{Shear and Compaction Bands}

When the modulus tensor, $L_{i j k l}$ (found by inverting (8)) is substituted into (2), the resulting expression can be solved for $H$, which varies with the constitutive parameters and the band orientation. Three band orientations are possible: shear bands $\left(n_{I I}=0\right)$, compaction bands $\left(n_{I I I}=1\right)$ and dilation bands $\left(n_{I}=1\right)$, where $n_{K}$ are the components of the unit vector normal to the band, and $K=I, I I, I I I$ are the principal stress directions. Rudnicki and Rice (1975), using $H=h=$ the slope of the shear stress-inelastic shear strain curve at constant 
mean stress, determined the expression for the critical value of the hardening modulus for shear bands

$$
\frac{h_{c r}^{I I}}{G}=\frac{1+\nu}{9(1-\nu)}(\beta-\mu)^{2}-\frac{1+\nu}{2}\left[N_{I I}+\frac{1}{3}(\beta+\mu)\right]^{2}
$$

where $G$ is the elastic (unloading) shear modulus and $\nu$ is Poisson's ratio. Since $h$ is expected to be monotonically decreasing with ongoing inelastic deformation, the expression (10) gives the first value of $h$ at which shear bands are possible if the following condition is satisfied (Perrin and Leblond, 1993):

$$
\begin{aligned}
(1-2 \nu) N_{I I}-\sqrt{4-3 N_{I I}^{2}} & \leq \frac{2}{3}(1+\nu)(\beta+\mu) \\
& \leq(1-2 \nu) N_{I I}+\sqrt{4-3 N_{I I}^{2}}
\end{aligned}
$$

where $N_{I I}=s_{I I} / \tau$, and $s_{I I}$ is the intermediate principal deviatoric stress.

When the left inequality of (11) is violated, compaction bands (perpendicular to the most compressive principal stress) are predicted, and the expression for the critical value of the hardening modulus is given by (Issen and Rudnicki , 2000)

$$
\begin{aligned}
\frac{h_{c r}^{I I I}}{G} & =\frac{1+\nu}{9(1-\nu)}\left\{(\beta-\mu)^{2}-\left[\frac{3}{2} N_{I I I}-(\beta+\mu)\right]^{2}\right\} \\
& -\left(1-\frac{3}{4} N_{I I I}^{2}\right)
\end{aligned}
$$

where $N_{I I I}=s_{I I I} / \tau$, and $s_{I I I}$ is the most compressive principal deviatoric stress. When the right inequality of (11) is violated, dilation bands are predicted (perpendicular to the least compressive principal stress), and the expression for the critical hardening modulus, $h_{c r}^{I}$, is found by replacing $N_{I I I}$ with $N_{I}=s_{I} / \tau$ in (12).

Perrin and Leblond (1993) noted that expressions (10) and (12) are continuous, such that as the left side of (11) approaches equality, the shear band angle (the angle between band normal and direction of maximum compression) approaches $0^{\circ}$, and the expressions for (10) and (12) are equal. Similarly, for the right side where the band angle approaches $90^{\circ}$. Additionally, Bésuelle (2000) showed that the band type varies smoothly from compaction bands to dilation bands through the range of (11).

The ranges of $\beta+\mu$ for which the solutions are valid are summarized in Figure 2 (for $\nu=0.2$ ). The upper and lower inequalities of (11) are shown as a function of $\sqrt{3} N_{I I}$. Shear bands are predicted in the central region, where (10) represents the largest critical value of the hardening modulus. Compaction bands are predicted for values of $\beta+\mu$ below the region, since (12) as written

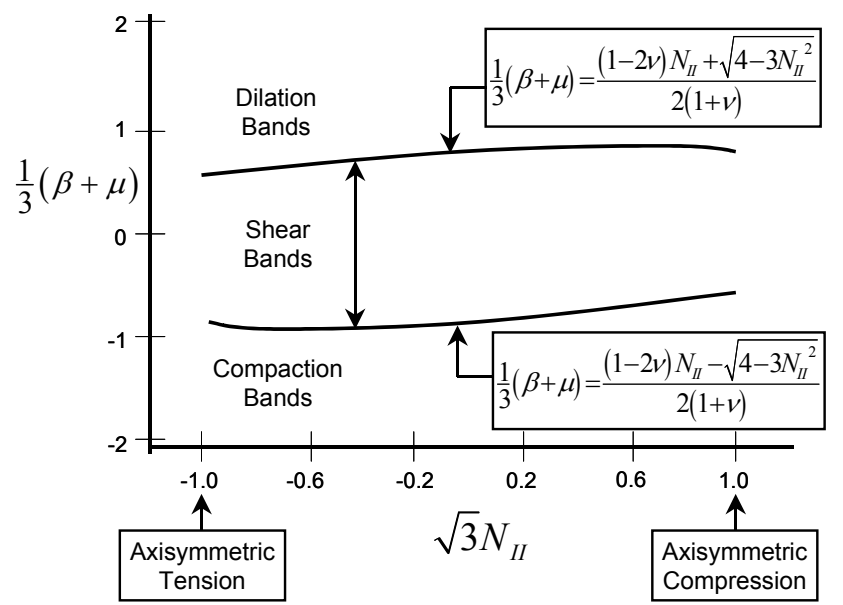

Fig. 2. Predicted band orientations as functions of $\beta+\mu$ and $N_{I I}$. Shear bands are predicted between the bold lines, while compaction bands are predicted below the lower line, and dilation bands are predicted above the upper line.

is greater than (12) with $N_{I}$ replacing $N_{I I I}$. Similarly, dilation bands are predicted above the central region.

Issen and Rudnicki (2000) note that axisymmetric compression is the most favorable stress state for compaction band formation, since (12) is largest for this stress state. For axisymmetric compression, $N_{I}=$ $N_{I I}=\sqrt{3}, N_{I I I}=-2 / \sqrt{3}$ and (12) reduces to

$$
\frac{h_{c r}^{I I I}}{G}=-\frac{1}{3} \frac{(1+\nu)}{(1-\nu)}\left(1+\frac{2}{\sqrt{3}} \mu\right)\left(1+\frac{2}{\sqrt{3}} \beta\right)
$$

Thus, compaction bands are predicted to form with positive $h_{c r}^{I I I}$ if $\mu<-\sqrt{3} / 2$ or $\beta<-\sqrt{3} / 2$ but not both. For axisymmetric compression, the condition on $\beta$ implies that the inelastic increment of lateral strain is compactive. Although the hardening modulus $h$ is not directly observable in axisymmetric compression, the slope of the stress difference - inelastic axial strain curve, $E_{p}$, is given by (Cleary and Rudnicki , 1976)

$$
E_{p}=9 h /(\sqrt{3}-\beta)(\sqrt{3}-\mu)
$$

If $\mu>\sqrt{3}$, the slope of the yield surface in $\tau$ vs. $\sigma$ exceeds the slope of the stress path for axisymmetric compression and hence inelastic deformation is not possible. If $\beta>\sqrt{3}$, the inelastic increment of axial strain must be extensile which contradicts the assumption of axisymmetric compression. Consequently, the sign of $E_{p}$ is the same as that of $h$ for the physically valid range of $\beta$ and $\mu$.

The observations of ? on Castlegate sandstone indicate that $E_{p}$ is positive and hence $h$ will also be positive. Figure 3 shows the regions of the $\beta$ vs. $\mu$ plane where compaction bands (fishscale) and shear bands (crosshatched) are predicted to occur with positive $h_{c r}$ and 


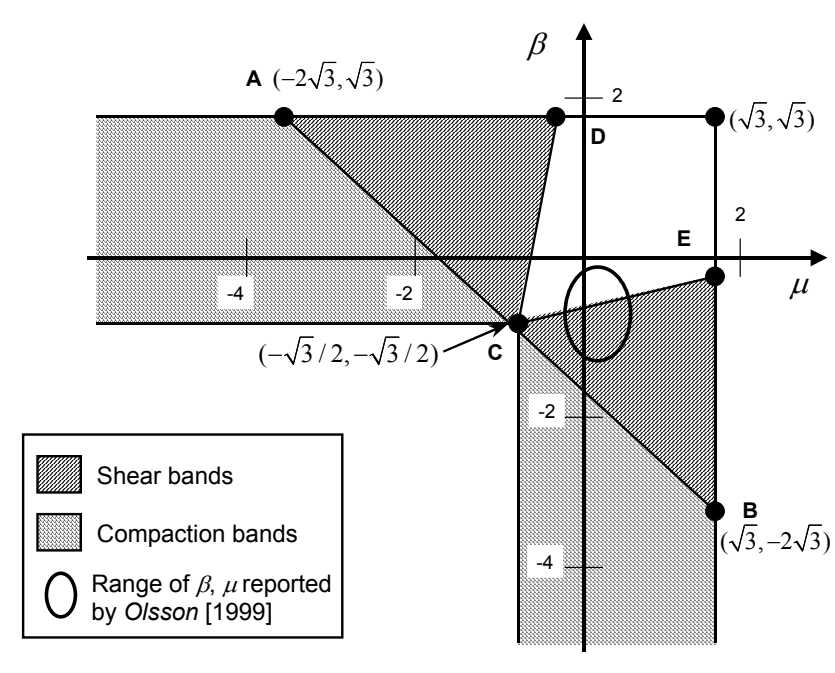

Fig. 3. Values of $\beta$ and $\mu$ where compaction bands (fishscale) and shear bands (cross-hatched) are predicted for axisymmetric compression with $h_{c r}>0$. Values reported by Olsson (1999) are within the oval. (Actual plot is for $\nu=0.2$ )

subject to the condition that both $\beta$ and $\mu$ be less than $\sqrt{3}$. The line $\mathrm{AB}$ corresponds to the left inequality in (11) and the lower boundary of the region for shear bands in Figure 2 for axisymmetric compression. A line through $\mathrm{DE}$ is given by

$$
\beta+\mu=\sqrt{3}\left(\sqrt{\frac{2}{(1-\nu}}-1\right) /\left(\sqrt{\frac{2}{(1-\nu}}+1\right)
$$

This value of $\beta+\mu$ is less than that given by right inequality in (11) for axisymmetric compression. Lines corresponding to $h_{c r}^{I I}=0$ are given by

$$
\beta=\left\{\mu\left(\sqrt{\frac{2}{(1-\nu}} \pm 1\right) \pm \sqrt{3}\right\} /\left(\sqrt{\frac{2}{(1-\nu}} \mp 1\right)
$$

where the upper sign applies for CD and the lower for CE. The values reported by ? (represented by the small oval in Figure 3) fall within (11). Therefore, only shear bands are predicted, but both shear and compaction bands were observed.

As noted earlier, the differing microscale mechanisms for dilatant and compactive deformation suggest the possibility of a yield surface comprising two facets, a shear yield surface and a "cap". If the loading path first intersects one facet, pushes it outward as hardening occurs, then intersects the second facet, both surfaces will be active. The case in which the loading path intersects the cap first (Wong et al. , 1992) is illustrated in Figure 4. If the facets meet smoothly, the results are the same as for the single active yield surface discussed above. If, however, the yield surfaces and plastic potentials form corners, the expressions for the values of

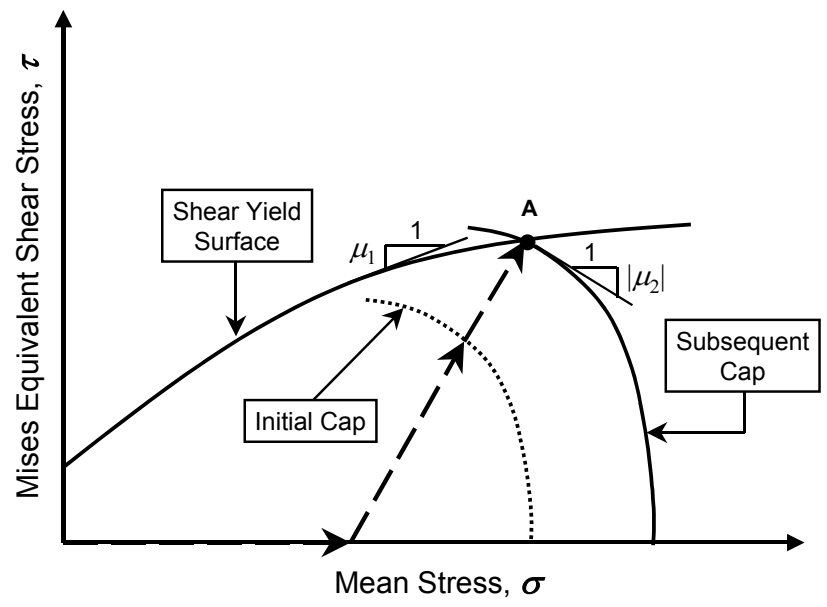

Fig. 4. A two yield surface model for high porosity rock, with an axisymmetric compression loading path that pushes the initial cap outward to the shear yield surface, thus activating both yield surfaces.

the critical hardening moduli become much more complicated. Nevertheless, simple results are possible for a few special cases.

As noted by Issen (2000), the modification of the constitutive relation by Aydin and Johnson (1983) corresponds to the introduction of a flat cap perpendicular to the mean stress axis. The approximate effect on the localization conditions (10) - (12) can be seen by replacing Poisson's ratio by an effective value $\nu^{*}$ :

$$
\nu^{*}=\frac{3 K^{*}-2 G}{2\left(3 K^{*}+G\right)}
$$

If $k<<K$, then $K^{*} \simeq k$, and $K^{*}<<G$, which results in $\nu^{*} \rightarrow-1$. As $\nu^{*} \rightarrow-1$, the critical hardening modulus for shear bands, $h_{c r}^{I I} \rightarrow 0$. For axisymmetric compression, the band angle, $\theta \rightarrow 0^{\circ}$, while $h_{c r}^{I I I} \rightarrow 0$. Thus, the Aydin and Johnson (1983) modification decreases the magnitude of the critical value of the hardening modulus at which localization is possible, and for a program of deformation where $h$ decreases with continued inelastic loading, compaction bands are predicted when $h=h_{c r}^{I I I}=0$.

It is interesting to note that Rudnicki and Rice (1975) showed that the effect of a yield surface vertex on the projection of the yield surface on the plane $\sigma=0$ could be treated approximately by introducing a deformation theory of plasticity. This had the effect of replacing the elastic shear modulus by a reduced effective value. Here, the effect of the Aydin and Johnson (1983) modification is to alter the effective value of Poisson's ratio.

Issen (2000) has also obtained simple results for the special case corresponding to $k=0$. High porosity sandstone under either pure hydrostatic compression or axisymmetric compression at "high" confining pressures is characterized by a stress - strain curve with a knee, 
followed by a mildly sloping region (shelf), where grain crushing and pore collapse occur (Zhang et al. , 1990; Wong et al. , 1992, 1997). For hydrostatic compression, the shelf corresponds to $k<K$, possibly $k<<K$. For axisymmetric compression, the slope is not strictly related to $k$ (since the shear stress is not constant), but $k<<K$ may be valid on the shelf and $k=0$ is a plausible first approximation. In this case, Issen (2000) showed that compaction bands are predicted for $h_{c r}^{I I I} \equiv 0$ in a wide range of values of dilatancy factors and friction coefficients (including those reported by ? for specimens with compaction bands) as long as the vertex formed by the two facets is convex.

\section{Conclusion}

We have summarized the predictions of Issen and Rudnicki (2000) for the occurrence of compaction bands using the approach of Rudnicki and Rice (1975) for shear bands. In particular, the appearance of the band is viewed as a bifurcation from, or alternative solution to, homogeneous deformation. In addition, we have discussed these results within a more general constitutive framework that includes the possibility of a vertex or corner where two facets of the yield surface intersect.

Unfortunately, definitive values of the constitutive parameters needed to evaluate the predictions carefully are not available. The values of the friction coefficient and dilatancy factor reported by ? at the formation of compaction bands are not sufficiently negative to agree with the theoretical predictions based on a single smooth yield surface. These values are, however, difficult to determine precisely at the onset of localization and can vary considerably with inelastic deformation (Holcomb and Rudnicki , 2000). Furthermore, results of examining a few special cases suggest that conditions for compaction bands may be considerably less stringent if the yield surface or plastic potential has a corner at the current load point. This seems likely to be the case for materials characterized by a shear surface and a cap under a range of loading conditions. Consequently, a more complete constitutive description in the vicinity of the intersect of the cap and shear surface is needed to contrain the predictions better.

Acknowledgements. Partial financial support for this work was provided by the U. S. Dept. of Energy, Office Basic Energy Sciences, Geosciences Research Program through Grant No. DEFG02-93ER14344/09 to Northwestern University. We are grateful to David Holcomb, Bill Olsson and Teng-fong Wong for many helpful discussions.

\section{References}

Aydin, A. and A.M. Johnson, Analysis of faulting in porous sandstones, J. Struct. Geol., 5, 10-31, 1983.
Bésuelle, P., Compacting and dilating shear bands in porous rock: theoretical and experimental conditions, submitted to J. Geophys. Res., 2000.

Cleary, M. P., and J. W. Rudnicki, The initiation and propagation of dilatant rupture zones in geological materials, in The Effects of Voids on Material Deformation (edited by S. C. Cowin) ASME Applied Mechanics Division, Vol. 16, [Proceedings of the Symposium on the Effects of Voids on Material Deformation, Salt Lake City, Utah, 14 June 1976], 13-30, 1976.

DiMaggio, F.L., and I. S. Sandler, Material model for granular Soils, J. Eng. Mech. Div., Am. Soc. Civ. Eng., 1971, 97, 935950, 1971.

Fossum, A.F., and J.T. Fredrich, Cap plasticity models and compactive and dilatant pre-failure deformation, Proc. 4 th North Am. Rock Mech. Symposium, 2000.

Hadamard, J., Leçons sur la Propagation de Ondes et les Equations de L'Hydrodynamique, Paris, 1903.

Hill, R., Acceleration waves in solids, J. Mech. Phys. Solids, 10, $1-16,1962$.

Hill, R. On constitutive inequalities for simple materials - I, $J$. Mech. Phys. Solids, 16, 229-242, 1968.

Holcomb, D. J. and J. W. Rudnicki, Inelastic constitutive properties and shear localization in Tennessee marble. Mechanics of Cohesive-Frictional Materials, in press, 2000.

Issen, K. A. and J. W. Rudnicki, Conditions for compaction bands in porous rock, J. Geophysical Res., 105, 21529-21536, 2000.

Issen, K.A., Conditions for localized deformation in compacting porous rock, Ph.D. Thesis, Northwestern University, Evanston, IL, 2000.

Mandel, J., Conditions de stabilté et postulat de Drucker, in Rheology and Soil Mechanics (ed. J. Kravtchenko and P. M. Sirieys) Springer-Verlag, pp. 58-68, 1966.

Menéndez, B., W. Zhu and T.-f. Wong, Micromechanics of brittle faulting and cataclastic flow in Berea sandstone, J. Struct. Geol., 18, 1-16, 1996.

Mollema, P. N. and M. A. Antonellini, Compaction bands: a sturctural analog for anti-mode I cracks in aeloian sandstone, Tectonophysics, 267, 209-228, 1996.

Perrin, G. and J. B. Leblond, Rudnicki and Rice's analysis of strain localization revisited, J. Appl. Mech., 60, 842-846, 1993.

Rudnicki, J. W. and J. R. Rice, Conditions for the localization of deformation in pressure-sensitive dilatant materials, J. Mech. Phys. Solids, 23, 371-394, 1975.

Rudnicki, J.W., and W.A. Olsson, Reexamination of fault angles predicted by shear localization theory, Int. J. Rock Mech. Min. Sci., 35, 4-5, 1998.

Thomas, T. Y., Plastic Flow and Fracture in Solids, Academic Press, 1961.

Wong, T.-f., H. Szeto and J. Zhang, Effect of loading path and porosity on the failure mode of porous rocks, Appl. Mech. Rev., 45, 281-293, 1992.

Wong, T.-f., C. David, and W. Zhu, The transition from brittle faulting to cataclastic flow in porous sandstones: mechanical deformation, J. Geophysical Res., 102, 3009-3025, 1997.

Zhang, J., T.-F., Wong, and D. M. Davis, Micromechanics of pressure-induced grain crushing in porous rock, J. Geophys. Res., 95, 341-352, 1990. 